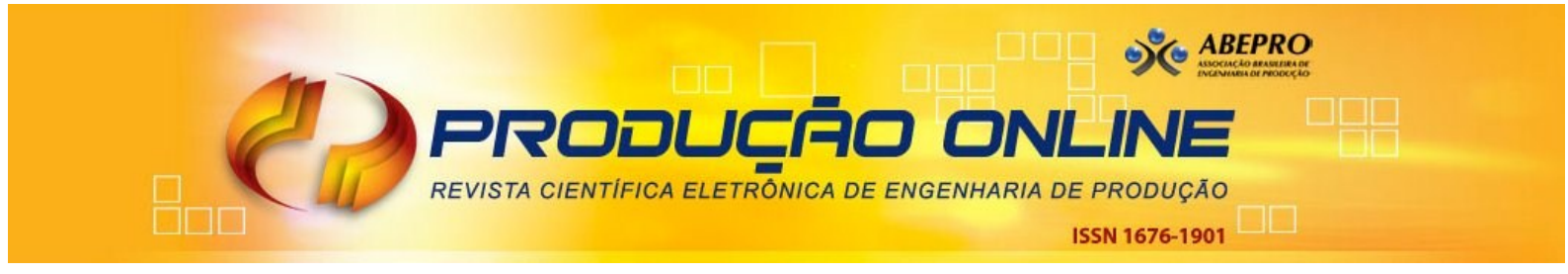

\title{
GRÁFICO EWMAREG APLICADO NO MONITORAMENTO DE PROCESSOS INDUSTRIAIS
}

\section{EWMAREG CONTROL CHART APPLIED IN THE MONITORING OF INDUSTRIAL PROCESSES}

\author{
Danilo Cuzzuol Pedrini* E-mail: danilo cp1@yahoo.com.br \\ Ângelo Márcio de Oliveira Sant'ana** E-mail: angelo.santanna@pucpr.br \\ Carla Schwengber ten Caten ${ }^{* * *}$ E-mail: tencaten@producao.ufrgs.br \\ *Petrobrás \\ ** Pontifícia Universidade Católica do Paraná, PUC/PR, Curitiba, PR \\ ***Universidade Federal do Rio Grande do Sul, UFRGS, Porto Alegre, RS
}

Resumo: Quando as características de qualidade do processo são dependentes das variáveis de controle e estas variam durante a execução do processo, violam-se as suposições necessárias à implementação dos gráficos de controle tradicionais. Se os valores das variáveis de controle do processo são conhecidos, é possível aplicar gráficos de controle de regressão. Um dos mais recentes gráficos desta linha é o EWMAREG, que consiste no monitoramento dos resíduos padronizados do modelo de regressão via gráfico de controle de médias móveis exponencialmente ponderadas. $O$ presente trabalho tem como objetivo apresentar uma sistemática para a aplicação do gráfico de controle EWMAREG no monitoramento de um processo simulado de uma indústria química. A característica monitorada foi a taxa de corrosão de tubos de aço, em função de quatro variáveis de controle. A ferramenta aplicada demonstrou elevado potencial de detectar alteração no comportamento da taxa de corrosão, garantindo a estabilidade do processo.

Palavras-chave: EWMAREG. Resíduos padronizados. Modelos de Regressão. Taxa de corrosão.

Abstract: If the process quality characteristics are dependent of control variables, and these vary during the process operation, the basic assumptions of control charts are violated. If the values of the control variables are known, it's possible to apply the regression control chart. One of the most recent works in this area is the EWMAREG chart, which is the monitoring of the standardized residuals using exponentially weighted moving average control chart. In this paper, we present a systematic application of the EWMAREG control chart in monitoring a simulated process of chemical industry. The process characteristic monitored was the corrosion rate of steel pipe in function of four process control variables. The tool applied demonstrated high potential to detect change in surveillance of corrosion rate, ensuring stability process.

Palavras-chave: EWMAREG; standardized residuals; regression models; corrosion rate

\section{INTRODUÇÃO}

Para a aplicação dos gráficos de controle tradicionais de Shewhart supõe-se que os dados monitorados sejam independentes e identicamente distribuídos em torno de uma média constante. Se essas suposições não forem verdadeiras, os gráficos de controle podem apresentar ineficiência na detecção de causas especiais

Revista Produção Online. Florianópolis, SC, v.11, n. 4, p. 1141-1159, out./dez. 2011. 
e altas taxas de alarmes falsos (MONTGOMERY, 2004; AIZENCHTADT ET al., 2008; RUSSO et al., 2008).

Tal suposição é violada quando ocorrem alterações frequentes nas variáveis de controle do processo, causando a alteração na média dos dados monitorados. Em outra situação, quando uma variável de controle é propositalmente ajustada para um valor extremo, a característica de qualidade pode assumir um valor diferente de seu comportamento usual, não havendo, no entanto, nenhuma falha no processo. Em ambos os casos, a característica de qualidade de um produto ou processo pode ser representada pelo seu relacionamento com as variáveis de controle, sendo possível o uso do gráfico de controle de regressão (JACOBI et al., 2002; LOREDO et al., 2002; SHU et al., 2007).

O GCR, proposto inicialmente por Mandel (1969), consiste no ajuste de um modelo de regressão linear simples relacionando a característica de qualidade do processo a uma variável de controle e o posterior monitoramento da característica de qualidade, comparada com o valor previsto pelo modelo de regressão. Segundo Mandel (1969), o GCR tem como principal função controlar uma variação média da característica de qualidade, acarretada pela ação das variáveis de controle do processo, ao invés de controlar uma média constante do processo como é usualmente feito nos demais gráficos de controle.

Segundo Pedrini e Caten (2010), a principal restrição ao GCR proposto por Mandel (1969) é a possibilidade de aplicação em processos que apresentem apenas uma variável de controle, o que inviabiliza a aplicação em vários processos industriais. Desta forma, autores como Zhang (1985), Hawkins (1991) e Haworth (1996) propuseram alternativas que permitem o monitoramento de processos que contenham mais de uma variável de controle, com o uso de modelos de regressão linear múltipla.

Tendo em vista a baixa sensibilidade dos procedimentos citados anteriormente em detectar pequenas alterações nos parâmetros do processo, Shu et al. (2004) propuseram o gráfico de controle EWMAREG, que consiste no monitoramento dos resíduos padronizados de um modelo de regressão linear múltipla (MRLM) usando um gráfico de controle de médias móveis exponencialmente ponderadas (EWMA). O trabalho elaborado por Shu et al. (2004) concentra-se na obtenção do número médio de amostras até o sinal (NMA) do 
gráfico de controle EWMAREG, sem apresentar uma orientação para a sua aplicação. Segundo Woodall (2000), esta é uma falha comum em grande parte dos trabalhos acadêmicos sobre gráficos de controle, o que dificulta a adoção dos novos gráficos de controle nos meios produtivos.

Assim, o presente trabalho tem como objetivo apresentar uma sistemática para a aplicação do gráfico de controle EWMAREG no monitoramento de um processo simulado de uma indústria química. A sistemática proposta consiste em duas fases de implantação para o gráfico de controle: análise retrospectiva (Fase I) e monitoramento do processo (Fase II).

\section{REVISÃO BIBLIOGRÁFICA}

\subsection{Modelos de Regressão Linear Múltipla}

A análise de regressão é uma técnica estatística que utiliza uma expressão matemática para representar a relação entre uma característica de qualidade e algumas variáveis de controle. De acordo com Montgomery et al. (2001) e Weisberg (2005), o modelo de regressão linear múltipla (MRLM) é representado pela equação (1):

$$
y=X \beta+\varepsilon
$$

onde $\mathbf{y}$ é o vetor dos valores da característica de qualidade, $\mathbf{X}$ uma matriz $(n \times k)$ composta pelos $n$ valores e $k$ variáveis de controle, $\boldsymbol{\beta}$ o vetor dos coeficientes de regressão e $\boldsymbol{\varepsilon}$ é o vetor dos erros aleatórios. O vetor dos erros aleatórios $\varepsilon$ é representado pelo estimador $\boldsymbol{e}$, obtidos através da diferença entre os valores observados e os valores estimados pelo modelo:

$$
e=y-\hat{y}
$$

Se o número de observações for maior que o número de variáveis de controle a serem incluídas no modelo, utiliza-se o método de mínimos quadrados ordinários (MQO) para estimar a equação de regressão (MONTGOMERY et al., 2001). estimador de mínimos quadrados ordinários de $\boldsymbol{\beta}$ é obtido pela resolução da equação (3).

$$
\hat{\beta}=\left(X^{\prime} X\right)^{-1} X^{\prime} y
$$

Para utilizar o MQO assume-se que os resíduos padronizados sejam independentemente distribuídos, com média igual a 0 e desvio-padrão constante. 
Esta suposição deve ser verificada após a construção do modelo, para a validação do modelo. Os valores estimados da variável resposta são representados por:

$$
\hat{y}=X \hat{\beta}=X\left(X^{\prime} X\right)^{-1} X^{\prime} y=H y
$$

Montgomery et al. (2001) e Weisberg (2005) comentam que a matriz H é chamada de matriz chapéu, pois transforma os valores observados y em valores estimados $\hat{y}$. Essa matriz é de grande importância no MRLM, principalmente na detecção de informações influentes e na estimativa do desvio-padrão para a previsão de uma nova observação.

\subsection{Gráfico de Controle de Regressão}

Em linhas gerais, o GCR é basicamente uma combinação dos gráficos de controle de Shewhart com a teoria de análise de regressão. A linha central (LC) do GCR é estimada pelo modelo de regressão da equação (1). A estimativa da variância do gráfico de controle de regressão é dada pela variância do modelo de regressão, estimado pelo quadrado médio dos resíduos (MANDEL, 1969).

onde $p=k+1$.

$$
\hat{\sigma}_{e}^{2}=\frac{e^{\prime} e}{n-p}=\frac{S Q R}{n-p}=Q M R
$$

De acordo com Mandel (1969), os limites de controle e linha central do gráfico de controle de regressão são dados por,

$$
\begin{gathered}
L S C_{i}=\hat{y}_{i}+2 \hat{\sigma}_{e} \\
L C_{i}=\hat{y}_{i} \\
L I C_{i}=\hat{y}_{i}-2 \hat{\sigma}_{e}
\end{gathered}
$$

Por sua vez, Haworth (1996) estendeu o procedimento clássico do GCR para monitorar processos que apresentem mais de uma variável de controle para explicar a característica de qualidade, mediante o monitoramento dos resíduos na forma student. Pedrini e Caten (2010) apresentam uma modificação ao GCR de Haworth (1996), permitindo o monitoramento direto da característica de qualidade ao invés do monitoramento dos resíduos, comparando também a sensibilidade do método proposto com outros procedimentos encontrados na literatura.

Rothschild e Roth (1986) e Loredo et al. (2003) propuseram a aplicação dos gráficos de controle de regressão para amostras individuais, utilizando a amplitude móvel dos resíduos ordinários para estimar o desvio-padrão para o cálculo dos Revista Produção Online. Florianópolis, SC, v.11, n. 4, p. 1141-1159, out./dez. 2011. 
limites de controle utilizado no gráfico. Zhang (1985) propôs o gráfico de controle para seleção de causas (GCSC), ferramenta aplicada no monitoramento de processos compostos por várias atividades sequenciais, também chamados de processos em cascata.

Expandindo a metodologia dos gráficos de controle de regressão, Olin (1998) utiliza modelos lineares generalizados (MLGs) e modelos não lineares para modelar a característica de qualidade. Skinner et al. (2003) e Jearkpaporn et al. (2003) propuseram o uso de gráficos de controle para resíduos deviance dos MLGs no monitoramento de contagem de não-conformidades com distribuição Poisson e Gama, respectivamente. Russo et al. (2008) usou o MLG para o monitoramento do número de não-conformidades em tecidos, seguindo uma distribuição Poisson.

Kang e Albin (2000) propõem o monitoramento de perfis lineares, através do monitoramento direto dos coeficientes de regressão e desvio-padrão estimados para cada nova amostra coletada. De acordo com Montgomery (2004), o GCR também pode ser utilizado para monitorar processos que apresentem tendências, que ocorrem devido à interferência de variáveis de controle do processo, como por exemplo, o desgaste de ferramentas.

\subsection{Gráfico de Controle EWMAREG}

Shu et al. (2004) propuseram o gráfico EWMAREG, que consiste no monitoramento dos resíduos padronizados de um modelo de regressão linear através de gráficos EWMA. Esta proposta, além de permitir o monitoramento de processos com mais de uma variável de controle, apresenta grande sensibilidade na detecção de pequenas variações no processo (SHU et al., 2004; PEDRINI e CATEN, 2009).

No método proposto por Shu et al. (2004), a característica de qualidade a ser amortizada exponencialmente é o resíduo padronizado de Pearson, apresentado na equação (9):

$$
z_{i}=\frac{e_{i}}{\hat{\sigma}_{e}}
$$

A variável monitorada no gráfico de controle é:

$$
U_{i}=\lambda z_{i}+(1-\lambda) U_{i-1}
$$

Revista Produção Online. Florianópolis, SC, v.11, n. 4, p. 1141-1159, out./dez. 2011. 
onde: $\lambda$ é a constante de amortização e $U_{0}=0$.

Os limites de controle para o gráfico de controle EWMAREG são iguais aos limites de controle dos gráficos EWMA, com a substituição da média por 0 , que é o valor esperado para a média dos resíduos de um modelo estimado pelo MQO. Estes limites são apresentados nas equações (11) e (13).

$$
\begin{aligned}
& L S C_{i}=+L \sqrt{\frac{\lambda}{2-\lambda}\left[1-(1-\lambda)^{2 i}\right]} \\
& L C=0 \\
& L I C_{i}=-L \sqrt{\frac{\lambda}{2-\lambda}\left[1-(1-\lambda)^{2 i}\right]}
\end{aligned}
$$

onde: $L$ é uma constante escolhida previamente.

\section{SISTEMÁTICA PROPOSTA}

A sistemática proposta no presente artigo foi adaptada a partir da sistemática para aplicação do GCR, apresentada por Pedrini e Caten (2010). A sistemática proposta foi estruturada em duas fases: Fase I - análise retrospectiva e Fase II monitoramento do processo, conforme sugestão apresentada por Faltin et al. (1997) e Woodall e Montgomery (1999).

Em linhas gerais, durante a Fase I de implantação dos gráficos de controle, realiza-se a análise de dados históricos do processo, com o objetivo de definir os parâmetros do gráfico de controle. Caso o processo seja considerado como estável, prossegue-se para a Fase II, que é o monitoramento do processo propriamente dito.

$\mathrm{Na}$ Fase II compara-se o resíduo, diferença entre a característica de qualidade observada e a estimada pelo modelo de regressão, com os limites de controle calculados a partir dos parâmetros obtidos na Fase I. Por ser aplicada diretamente pelos operadores do processo, para a Fase II sugere-se que sejam realizados treinamentos específicos de coleta de dados, inserção de valores em planilhas e interpretação dos resultados dos gráficos de controle apresentados.

Para a aplicação da sistemática proposta são necessárias duas suposições: (i) a característica de qualidade do processo deve ser uma variável contínua e sua relação com as variáveis de controle deve ser ajustável por um modelo de regressão linear e (ii) os resíduos do modelo de regressão devem ser independentemente e normalmente distribuídos, com média 0 e desvio-padrão aproximadamente 
constante. A suposição de normalidade dos resíduos é necessária para a realização de inferências sobre os coeficientes de regressão e estimativas da característica de qualidade (MONTGOMERY et al., 2001; PEDRINI e CATEN, 2010).

\subsection{Fase I - Análise Retrospectiva}

A Fase I da sistemática proposta apresenta três etapas: (i) coleta de dados históricos, (ii) ajuste do modelo de regressão linear múltipla e (iii) construção do gráfico de controle EWMAREG para a Fase I.

\subsubsection{Coleta de Dados}

Para estimar o modelo de regressão que descreve o relacionamento entre a característica de qualidade do processo e as variáveis de controle, é necessário coletar uma amostra significativa do processo. Assim, é possível estimar todos os coeficientes de regressão, além de disponibilizar o maior número possível de graus de liberdade para o termo de erro do modelo, o que aumenta a confiabilidade dos resultados. Ressalta-se que os dados coletados devem conter o valor da característica de qualidade monitorada e os valores das variáveis de controle do processo referentes ao ajuste do processo no momento de coleta.

\subsubsection{Ajuste do Modelo de Regressão}

O modelo de regressão linear múltipla é ajustado através do método MQO que estima os coeficientes do modelo de regressão. $O$ uso do MQO pressupõe que o número de amostras $(\mathrm{m})$ deve ser maior que o número de variáveis de controle $(\mathrm{k})$ mais um (MONTGOMERY et al., 2005).

Após a estimativa do modelo de regressão, sugerem-se seis passos para a aceitação do modelo: (i) teste de significância do modelo de regressão; (ii) uso do fator de inflação da variância (FIV) para a análise de ocorrência de multicolinearidade no modelo; (iii) teste dos coeficientes individuais de regressão, de forma a verificar a significância da relação entre cada uma das variáveis de controle e a característica de qualidade; (iv) uso da distância de Cook para análise de pontos 
influentes na estimativa do modelo; ( $v$ ) análise das suposições de que os resíduos são normalmente distribuídos com média zero e desvio-padrão aproximadamente constante e (vi) uso da estatística de Durbin-Watson para verificar a hipótese de que os resíduos são independentes. Para maiores detalhes sobre estes passos, sugerese Montgomery et al. (2001) e Weisberg (2005).

\subsubsection{Gráfico de controle EWMAREG da Fase I}

Após a validação do modelo de regressão estimado, é possível estimar os valores da característica de qualidade, dados os valores das variáveis de controle. Para a Fase I da sistemática proposta, a característica de qualidade monitorada pelo gráfico de controle passa a ser o resíduo padronizado, que pode ser obtido através da equação (9).

Para definir os valores das constantes $L$ e $\lambda$ do gráfico de controle EWMAREG, é necessário definir: (i) taxa de ocorrência de alarmes falsos; (ii) alteração na média dos resíduos que se deseja detectar. Para maiores detalhes sobre a escolha dos valores das constantes $\lambda$ e L, sugere-se a consulta aos trabalhos de Crowder (1987; 1989), Lucas e Saccucci (1990) e Shu et al. (2004).

Os limites de controle e linha central da Fase I foram dados nas equações (11), (12) e (13). É importante destacar que estes limites não são constantes, sendo estreitos no início do procedimento, se alargando à medida que se aumenta o número de amostras coletadas e, a partir de uma amostra $i$ muito grande, estes limites serão praticamente constantes (MONTGOMERY, 2004).

Caso alguma amostra exceda os limites de controle, o processo será considerado como estando fora de controle estatístico e, dessa forma, as causas especiais que alteraram o estado do processo deverão ser investigadas. Se forem detectadas as causas especiais que geraram os pontos fora de controle, o modelo de regressão e os limites de controle deverão ser calculados novamente, desconsiderando-se os pontos fora de controle. Caso não existam novos pontos fora de controle, procede-se para a Fase II da sistemática proposta. 


\subsection{Fase II - Monitoramento do Processo}

Em linhas gerais, a Fase II é o monitoramento do processo, sendo subdividido em três etapas de execução: (i) coleta de dados, (ii) monitoramento das variáveis de controle e (iii) monitoramento da característica de qualidade. Segundo Faltin et al. (1997), para a aplicação da Fase II é necessário supor que os dados monitorados e os dados utilizados na Fase I sigam a mesma distribuição. Para o gráfico de controle EWMAREG, isto implica na possibilidade de utilizar o modelo de regressão e o desvio-padrão do modelo para gerar a previsão da característica de qualidade e dos valores dos resíduos padronizados.

\subsubsection{Coleta de Dados}

Para a Fase II de aplicação do gráfico de controle EWMAREG coletam-se, em intervalos regulares de tempo, amostras individuais do processo. Assim como na Fase I, os dados coletados para o monitoramento do processo devem conter o valor da característica de qualidade monitorada e respectivos valores das variáveis de controle do processo.

\subsubsection{Monitoramento das Variáveis de Controle}

Antes do monitoramento da característica de qualidade, é necessário verificar se os valores das variáveis de controle referentes a esta amostra estão extrapolando a região original definida pelo intervalo de investigação do conjunto de variáveis de controle utilizadas para estimar o modelo de regressão. Esse passo é importante, pois a extrapolação pode gerar uma estimativa ruim para a característica de qualidade, prejudicando, conseqüentemente, o desempenho do gráfico de controle EWMAREG (MONTGOMERY et al., 2001; WEISBERG, 2005). Para verificar se ocorre extrapolação de cada amostra coletada, utiliza-se o gráfico de controle de extrapolação, proposto por Pedrini e Caten (2010), que é uma adaptação gráfica de um procedimento para verificação de extrapolação apresentado em Montgomery et al. (2001).

Segundo Pedrini e Caten (2010), a variável monitorada pelo gráfico de controle de extrapolação é o elemento $h_{\mathrm{j} j}$, apresentado na equação (14). 


$$
h_{j j}=x_{j}^{\prime} \cdot\left(X^{\prime} X\right)^{-1} \cdot x_{j}
$$

onde $x_{j}^{\prime}$ é o vetor das variáveis de controle da $j$-ésima observação; $\boldsymbol{X}$ é uma matriz definida por todos os vetores de valores das variáveis de controle utilizadas para estimar o modelo de regressão.

Este gráfico apresenta somente o LSC, que é igual ao maior valor de $h_{i i}$ dentre todos os vetores $x_{i}$ utilizados para estimar o modelo de regressão da Fase $\mathrm{I}$.

Caso algum $h_{j j}$ exceda o LSC, está ocorrendo a extrapolação do intervalo de investigação das variáveis de controle do modelo de regressão e as causas especiais que geraram esta amostra fora de controle devem ser identificadas. Especificamente para o gráfico de controle de extrapolação, um ponto fora de controle pode indicar: (i) erro de ajuste do processo por parte dos operadores; (ii) erro de coleta de dados ou (iii) alteração nos parâmetros de ajuste do processo, indicando a necessidade de reinício da Fase I do método proposto (PEDRINI e CATEN, 2010).

\subsubsection{Monitoramento da Característica de Qualidade}

Para a Fase II do GCR, considera-se que o valor previsto pelo modelo de regressão é o valor ideal da característica de qualidade do processo, dados os valores das variáveis de controle (PEDRINI e CATEN, 2010). No caso do gráfico de controle EWMAREG, esperam-se resíduos próximos a zero, o que indica que o valor observado da característica de qualidade está próximo do valor estimado pelo modelo de regressão.

Por se tratar de uma nova amostra, considerada independente das amostras utilizadas para estimar o modelo de regressão, é necessário corrigir o desvio-padrão da previsão de uma nova observação para cada amostra em relação ao afastamento do novo vetor de variáveis de controle em relação ao valor médio de cada variável de controle da Fase I (HAWORTH, 1996; MONTOMERY et al., 2010). Para isso, utiliza-se o elemento $h_{\mathrm{jj}}$, apresentado na equação Erro! Fonte de referência não encontrada., que é calculado com vetor $x_{j}$ coletado. A partir da correção no desviopadrão, altera-se o cálculo dos resíduos padronizados, gerando-se os resíduos na forma student, apresentados na equação (15).

$$
s_{j}=\frac{e_{j}}{\hat{\sigma} \sqrt{1+h_{j j}}}
$$

Revista Produção Online. Florianópolis, SC, v.11, n. 4, p. 1141-1159, out./dez. 2011. 
A variável monitorada pelo gráfico EWMAREG é obtida pela equação (16).

$$
U_{j}=\lambda s_{j}+(1-\lambda) U_{j-1}
$$

Os limites de controle para a Fase II do gráfico EWMAREG são adaptados a partir dos limites de controle da Fase I, considerando-se que o índice $j$ é um número muito grande. Dessa forma, os limites de controle serão fixos e paralelos à linha central.

$$
\begin{array}{r}
L S C=+L \sqrt{\frac{\lambda}{2-\lambda}} \\
L C=0 \\
L I C=-L \sqrt{\frac{\lambda}{2-\lambda}}
\end{array}
$$

Ressalta-se que para o gráfico de controle EWMAREG, o valor dos resíduos fora dos limites de controle indica, necessariamente, uma diferença incomum entre o valor observado da característica de qualidade e o valor estimado pelo modelo de regressão. Neste caso, deve-se proceder com a investigação das causas especiais que levaram a este valor incomum.

\section{Aplicação da Sistemática Proposta}

A sistemática proposta foi aplicada em um processo simulado de uma indústria química, cuja característica de qualidade a ser monitorada é a taxa de corrosão em tubulações de aço carbono, em função de variáveis de controle do processo: temperatura do banho químico $\left(\mathrm{x}_{1}\right) ; \mathrm{pH}$ do fluido $\left(\mathrm{x}_{2}\right)$; dosagem de anticorrosivo $\left(x_{3}\right)$ e vazão do fluido $\left(x_{4}\right)$.

O monitoramento da corrosão destas tubulações de aço carbono pode ser realizado por meio de provadores de corrosão e de sondas corrosimétricas. Este monitoramento é de grande importância para as indústrias químicas, pois é possível evitar acidentes de trabalho e ambientais.

O uso do gráfico de controle de regressão aplicado ao monitoramento da corrosão visa identificar quais variáveis de controle do processo influenciam a corrosão, prever a corrosão das tubulações dado o ajuste das variáveis de controle de operação do processo e identificar momentos de ocorrência de taxas de corrosão que divirjam do previsto pelo modelo. 


\subsection{Aplicação da Fase I}

Os dados utilizados para a Fase I foram obtidos através de um planejamento experimental realizado na indústria química estudada. O modelo de regressão, estimado pelo método de mínimos quadrados ordinários, é apresentado na equação (21).

$$
\hat{y}=90,38+9,50 x_{1}-5,13 x_{2}-6,48 x_{3}+12,00 x_{4}-8,21 x_{1}^{2}
$$

Este modelo apresenta um coeficiente de determinação de aproximadamente $87,3 \%$ e um coeficiente de determinação ajustado de 83,9\%. Para testar a significância do modelo de regressão estimado, utiliza-se o teste $F$, calculada através da análise de variância (ANOVA) da regressão, que é apresentada na Tabela 1.

Tabela 1 - ANOVA para o modelo estimado.

\begin{tabular}{cccccc}
\hline Fonte & SQ & GL & QM & F & Valor-p \\
\hline Regressão & 9180,88 & 5 & 1836,17 & 21,83 & 0,00 \\
Erro & 1598,25 & 19 & 84,12 & & \\
Total & 10779,13 & 24 & & & \\
\hline
\end{tabular}

O teste de significância do modelo de regressão apresentou um valor-p menor que o nível de significância adotado $(5 \%)$ e, assim, conclui-se que há fortes evidências para considerar que a taxa de corrosão seja linearmente dependente de pelo menos uma das variáveis de controle selecionadas no modelo. Na Tabela 2, apresenta-se o fator de inflação de variância (FIV), para verificar a presença de multicolinearidade no modelo, e os resultados dos testes individuais para os coeficientes de cada variável de controle e do intercepto.

Tabela 2 - Testes t individuais e valores FIV para as variáveis de controle e coeficiente de intercepto.

\begin{tabular}{cccccc}
\hline . Termo & Coeficiente & Desvio-padrão & T & Valor-p & FIV \\
\hline Intercepto & 90,381 & 2,30 & 39,34 & 0,000 & - \\
$x_{1}$ & 9,496 & 1,69 & 5,62 & 0,000 & 1,0 \\
$x_{2}$ & $-5,129$ & 1,69 & $-2,73$ & 0,007 & 1,0 \\
$x_{3}$ & $-6,479$ & 1,69 & $-3,46$ & 0,001 & 1,0 \\
$x_{4}$ & 12,004 & 1,69 & 6,72 & 0,000 & 1,0 \\
$x_{1}^{2}$ & $-8,213$ & 1,66 & $-4,33$ & 0,000 & 1,0 \\
\hline
\end{tabular}

Na Tabela 2 observa-se que os valores do FIV de todas variáveis incluídas no modelo foram menores do que 5 , logo não há multicolinearidade entre as variáveis de controle. Os testes estatísticos $t$-student para o coeficiente de intercepto e os das Revista Produção Online. Florianópolis, SC, v.11, n. 4, p. 1141-1159, out./dez. 2011. 
variáveis de controle apresentam valor-p menores que $5 \%$, sendo considerados significativos. Para validação do modelo, apresenta-se na Figura 1, o gráfico de resíduos padronizados versus valores estimados do modelo.

Figura 1 - Gráficos dos resíduos para validação do modelo.

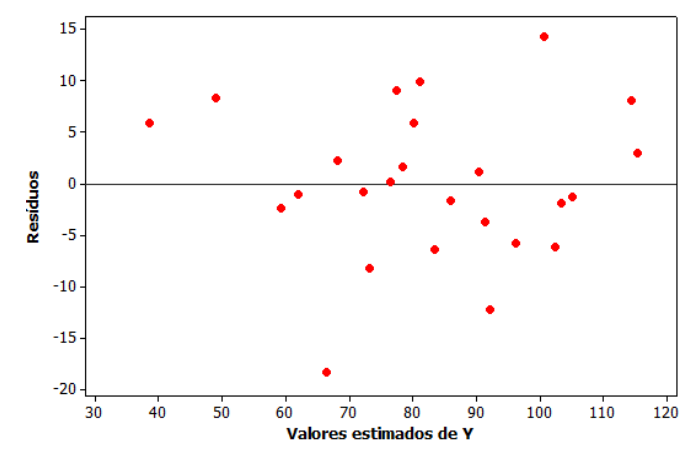

Através da análise da Figura 1, observa-se que a variabilidade dos resíduos pode ser considerada como sendo aproximadamente constante, já que esta se aproxima do padrão descrito por Montgomery et al. (2001), não apresentando evidências de que a variabilidade sofre variações com a alteração do valor estimado pelo modelo. O resultado do teste de normalidade Kolmorov-Smirnov apresentou um valor- $p$ de aproximadamente 0,15 , demonstrando que os resíduos padronizados podem ser considerados como normalmente distribuídos.

Para a verificação de independência dos resíduos, as estatísticas $d_{p}$ e $d_{n}$ do teste de Durbin-Watson foram de 2,07 e 1,93, respectivamente. Como estes valores foram superiores ao valor crítico tabelado, para um nível de significância de 5\% $(1,886)$, conclui-se que os resíduos sejam indepententes. Desta forma, se valida o modelo de regressão múltipla estimado.

O modelo de regressão estimado, além de servir como base para a elaboração do gráfico de controle proposto, ainda tem a utilidade de permitir prever a corrosão dado o ajuste das variáveis de controle de operação da planta química, possibilitando identificar um melhor ajuste do processo para minimizar a taxa de corrosão.

Como o modelo estimado foi validado, utiliza-se o gráfico de controle EWMAREG da Fase I para verificação de estabilidade dos dados utilizados para estimar o modelo de regressão linear múltipla. Para este gráfico, adotou-se $L=2,71$ e $\lambda=0,15$, conferindo um $\mathrm{NMA}_{0}$ de aproximadamente 370 (CROWDER, 1987).

Revista Produção Online. Florianópolis, SC, v.11, n. 4, p. 1141-1159, out./dez. 2011. 
Adotando estes valores para as constantes, os limites de controle da Fase I são dados pelas equações (21) e (22).

$$
\begin{aligned}
& L S C_{i}=+0,797 \sqrt{(1-0,85)^{2 i}} \\
& L I C_{i}=-0,797 \sqrt{(1-0,85)^{2 i}}
\end{aligned}
$$

Adotando estes , o gráfico EWMAREG para o monitoramento dos resíduos padronizados é apresentado na Figura 2.

Figura 2 - Gráfico de controle EWMAREG da Fase I

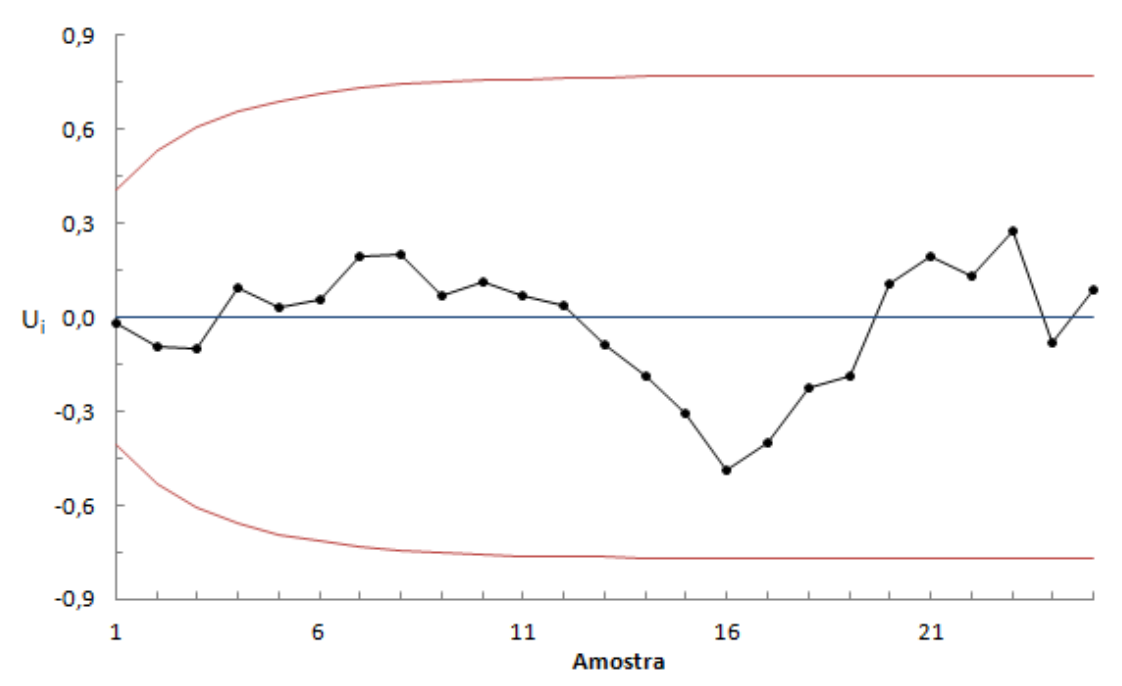

Como o gráfico EWMAREG, apresentado na Figura 2, não apresentou nenhum ponto fora de controle, o processo é considerado como estando sob controle estatístico. Dessa forma, o modelo de regressão apresentado na equação (21) e o desvio-padrão do processo podem ser utilizados para monitorar a taxa de corrosão do processo pesquisado, podendo avançar para a Fase II da sistemática proposta.

\subsection{Aplicação da Fase II}

Em seguida, inicia-se a Fase II da sistemática proposta, onde foram utilizadas 80 novas amostras provenientes do processo químico estudado, coletadas pelos operadores do processo. Após a coleta dos dados, utiliza-se o gráfico de controle de extrapolação para verificar se os valores do conjunto de variáveis de controle extrapolam a região formada pelos intervalos de investigação utilizados para estimar 
o modelo de regressão. O LSC encontrado para este gráfico é igual a 0,577. O gráfico de controle de extrapolação é apresentado na Figura 3:

Figura 3 - Gráfico de controle de extrapolação

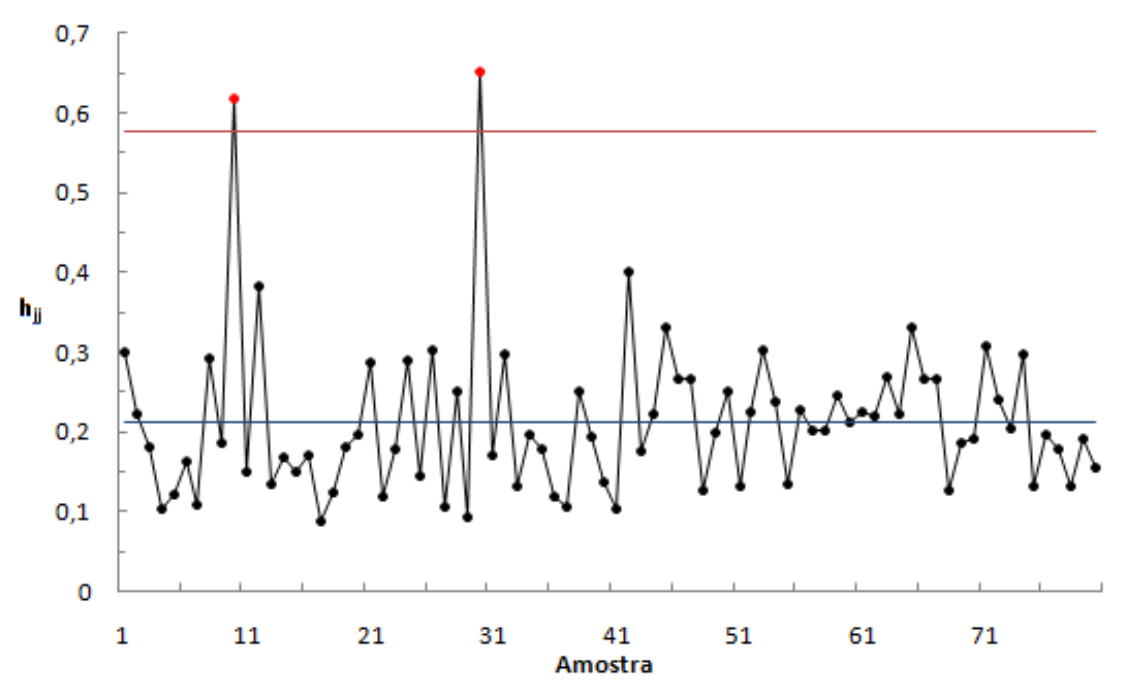

Como é possível observar na Figura 3, as amostras 10 e 30 excederam o LSC do gráfico de controle de extrapolação e serão desconsideradas do monitoramento do gráfico de controle de regressão múltipla. Neste caso, apontaramse erros de coleta como a causa do problema. Após a exclusão desta amostra restaram 78 amostras que foram monitoradas pelo gráfico de controle de EWMAREG. Adotando o valor do QMR do modelo estimado, os resíduos padronizados na forma student podem ser obtidos através da sequinte equação:

$$
s_{j}=\frac{e_{j}}{8,06 \sqrt{1+h_{j j}}}
$$

Os limites de controle para o gráfico EWMAREG da Fase II são apresentados nas equações (24) e (25), adotando-se os mesmos valores das constantes $L$ e $\lambda$ da Fase I.

$$
\begin{aligned}
L S C & =+0,797 \\
L I C & =-0,797
\end{aligned}
$$

A Figura 4 apresenta o gráfico de controle EWMAREG da Fase II para o monitoramento da taxa de corrosão de tubos de aço-carbono, utilizando os resíduos na forma student e os limites de controle apresentados nas equações (24) e (25). 
Figura 4 - Gráfico de controle EWMAREG da Fase II

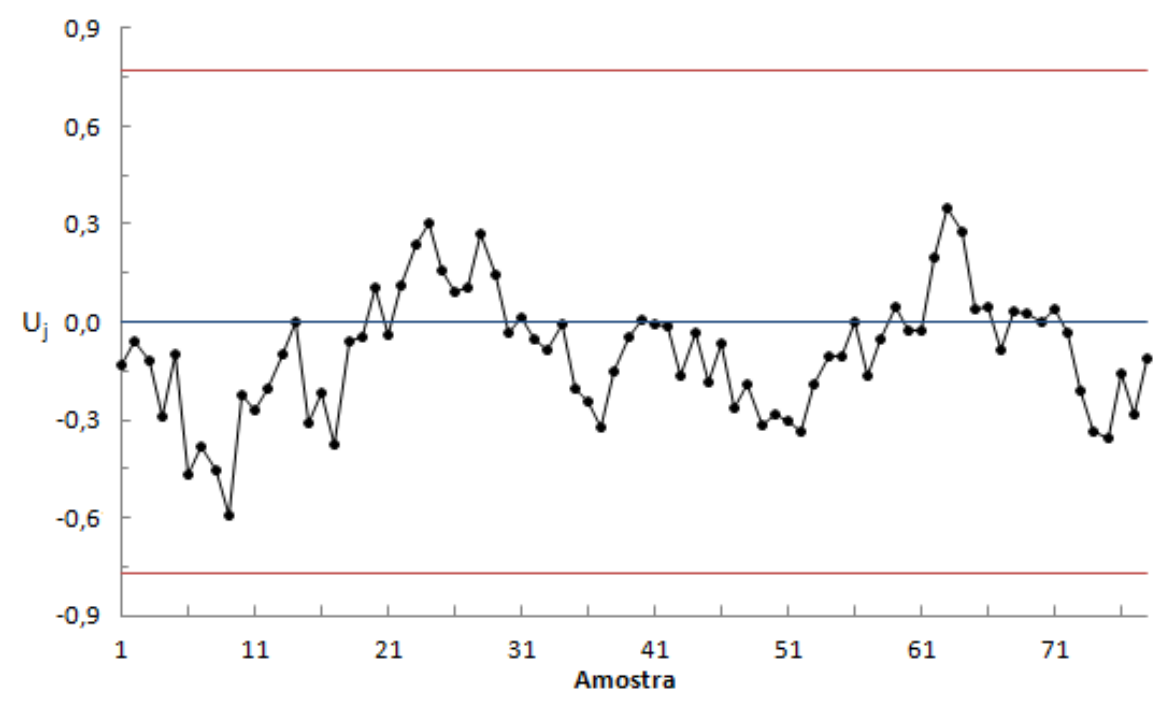

Analisando a Figura 4, é possível concluir que o processo está sob controle estatístico, já que o gráfico de controle proposto não indicou nenhuma amostra como fora da região delimitada pelos limites de controle.

\section{CONCLUSÕES}

O presente trabalho teve como objetivo apresentar uma sistemática para aplicação do gráfico de controle EWMAREG, sendo exemplificada pelo monitoramento da taxa de corrosão de tubos de aço em um processo industrial. A sistemática foi estruturada em duas fases inter-relacionadas: Fase I - análise retrospectiva - e Fase II - monitoramento do processo propriamente dito, sendo detalhadas as etapas de cada fase.

A Fase I da sistemática proposta inclui as etapas de: coletas de dados, ajuste do modelo de regressão linear múltipla e a verificação da estabilidade do processo através do gráfico de controle EWMAREG da Fase I. Se o processo for considerado como estando sob controle estatístico e, assumindo que o modelo de regressão linear múltipla estimado esteja adequado, prossegue-se à Fase II da sistemática proposta. A Fase II foi subdividida nas seguintes etapas: coleta de novas amostras individuais do processo, monitoramento das variáveis de controle (com o uso do gráfico de controle de extrapolação) e monitoramento da característica de qualidade, através do gráfico de controle EWMAREG da Fase II.

Revista Produção Online. Florianópolis, SC, v.11, n. 4, p. 1141-1159, out./dez. 2011. 
Os gráficos de controle EWMAREG das duas fases são conceitualmente similares, mas apresentam duas diferenças. A primeira ocorre pelo uso de limites de controle constantes no gráfico da Fase II e de limites de controle variáveis no gráfico da Fase I, que se alargam à medida que aumenta o número da amostra coletada. A segunda diferença ocorre no uso do resíduo padronizado na Fase I como variável a serem analisadas e do uso dos resíduos na forma student na Fase II, que ocorre devido à inclusão do erro de previsão de novas observações do modelo de regressão.

A sistemática foi aplicada e validada em um processo químico simulado, sendo monitorada a taxa de corrosão de tubos de aço-carbono em função de quatro variáveis de controle (temperatura; $\mathrm{pH}$ do fluido; dosagem de anti-corrosivo e vazão do fluido). Nesta aplicação explicitaram-se os passos intermediários das fases do método proposto, de forma a ressaltar a facilidade que esta sistematização pode gerar na aplicação.

Embora a aplicação do gráfico EWMAREG não tenha indicado a ocorrência de pontos fora de controle, o uso desta ferramenta tem o potencial de apontar momentos em que a taxa de corrosão apresente comportamento diferente do esperado. $\mathrm{O}$ uso do modelo de regressão estimado além de gerar os parâmetros para o ajuste do gráfico de controle de regressão, ainda possibilita prever a taxa de corrosão e ajustar as variáveis de controle do processo para minimizar a taxa de corrosão.

\section{REFERÊNCIAS}

CROWDER, S. V. A Simple method for studying run-length distributions of exponentially weighted moving average charts. Technometrics, v. 29, n. 4, p. 401407, 1987.

CROWDER, S. V. Design of exponentially weighted moving average schemes. Journal of Quality Technology, v. 21, n. 3, p. 155-162, 1989.

EIZENCHTADT, E.; INGMAN, D.; FRIEDLER, E. Quality control of wastewater treatment: a new approach. European Journal of Operations Research, v. 189, p. 445-458, 2008.

FALTIN, F. W.; MASTRANGELO, C. M.; RUNGER, G. C.; RYAN, T. P. Considerations in the monitoring of autocorrelated and independent data. Journal of Quality Technology, v. 29, n. 2, p. 131-133, 1997. 
HAWKINS, D. M. Multivariate quality control based on regression-adjusted variables. Technometrics, v. 33, n.1, p. 61-75, 1991.

HAWORTH, D. A. Regression control chart to manage software maintenance. Software Maintenance: Research and Practice, v. 8, n. 1, p 35-48, 1996.

JACOBI, L. F.; SOUZA, A. M.; PEREIRA, J. E. S. Gráfico de controle de regressão aplicado na monitoração de processos. Revista Produção, v. 12, n. 1, p. 46-59, 2002.

JEARKPAPORN, D.; MONTGOMERY, D. C.; RUNGER, G. C.; BORROR, C. M. Process monitoring for correlated gamma-distributed data using generalized-linearmodel-based control charts. Quality and Reliability Engineering International, v. 19, n. 6, p. 477-491, 2003.

KANG, L.; ALBIN, S. On-line monitoring when the process yelds linear profiles. Journal of Quality Technology, v. 32, n. 4, p. 418-426, 2000.

LOREDO, E. N.; JEARKPAPORN, D.; BORROR, C. M. Model-based control chart for autoregressive and correlated Data. Quality and Reliability Engineering International, v. 18, n. 6, p. 489-496, 2002.

LUCAS, J. M.; SACCUCCI, M. S. Exponentially weighted moving average control schemes: Properties and Enhancements. Technometrics, v. 32, n.1, p. 1-12, 1990.

MANDEL, B. J. The regression control chart. Journal of Quality Technology, v. 1, n. 1, p. 1-9, 1969.

MONTGOMERY, D. C. Introdução ao controle estatístico da qualidade. 4. ed. Rio de Janeiro: Editora LTC, 2004, 513 p.

MONTGOMERY, D. C.; VINING, G. G.; PECK, E. A. Introduction to linear regression analysis. 3. Ed., New York: John Wiley \& Sons, 2001, 641 p.

OLIN, B. D. Regression control charts revisited: methodology and cases studies. New York: $42^{\circ}$ Annual Fall Technical Conference (AFTC), 1998.

PEDRINI, D. C; CATEN, C. S. Método para a aplicação de gráficos de controle de regressão no monitoramento de processos, 2010. (Aprovado para publicação na Revista Produção).

ROTHSCHILD, B. F.; ROTH, S. R. Statistical process control of plating solutions with regression control charts. The SAMPE Journal, v. 22, n. 5, p. 37-41, 1986.

RUSSO, S. L.; CAMARGO, M. E.; SAMOHYL, R. W. Gráfico de controle baseado nos resíduos do modelo de regressão Poisson. Produção Online, v. 8, n. 4, 11 p.. 2008. 
SHU, L; TSUNG, F; TSUI, K. L. Run-length perfomance of regression control charts with estimated parameters. Journal of Quality Technology, v. 36, n. 3, p. 280-292, 2004.

SKINNER, K. R.; MONTGOMERY, D. C.; RUNGER, G. C. Process monitoring for multiple count data using generalized linear model-based control charts.

International Journal of Production Research, v. 41, n. 6, p. 1167-1180, 2003.

WEISBERG, S. Applied linear regression. 3. ed. Nova lorque: John Wiley \& Sons, 2005, $310 \mathrm{p}$.

WOODAL, W. H. Controversies and contradictions in statistical process control. Journal of Quality Technology, v. 32, n. 4, p. 341-350, 2000.

WOODAL, W. H.; MONTGOMERY, D.C. Research issues and ideas in statistical process control. Journal of Quality Technology, v. 31, n. 4, p. 376-386, 1999.

ZHANG, Z. X. Cause-selecting control charts : a new type of quality control charts. The QR Journal, v. 12, p. 221-225, 1985.

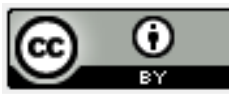

Artigo recebido em 20/09/2010 e aceito para publicação em 30/09/2011. 